\title{
Multiscale Modeling for the Simulation of Damage Processes at Refractory Materials under Thermal Shock
}

\author{
D. Henneberg, A. Ricoeur
}

A brittle material damage model based on the theoretical concept of continuum damage mechanics is presented. Cell models are developed including microcrack initiation and growth. To combine fractureand damage-mechanical approaches, submodels containing a sharp crack tip are introduced at the ends of the damage zones. Also, a conservation integral is applied yielding the energy release rate of an equivalent macro-crack.

\section{Introduction \\ Refractories are generally subjected to com- bined thermomechanical loading. The chal- lenging aim in this research field is to devel- op refractory structures with materials prop- erties matched for specific applications. Above all, the thermal shock resistance is the one mechanical property, which has to be improved. This requires an understanding}

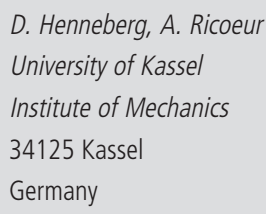

Received: 06.10.2011

Accepted: 24.10.2011

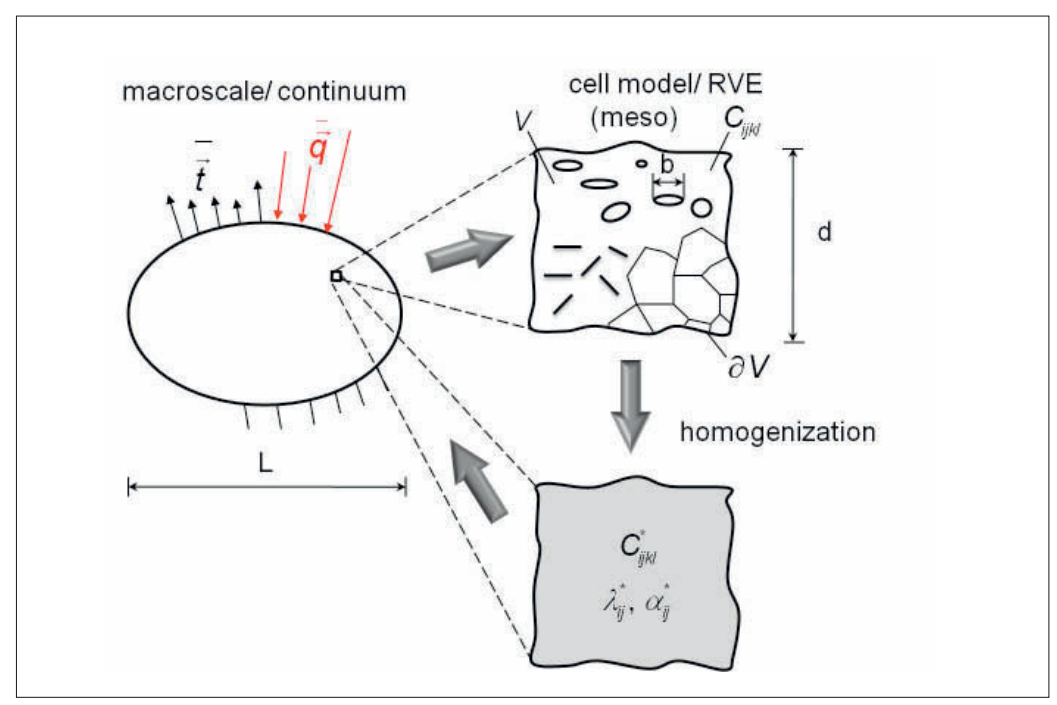

Fig. 1 Problem formulation on different scales and concept of homogenization $\left(C_{i j k l}^{*}\right.$ : effective elastic tensor, $\lambda_{i j}^{*}$ effective thermal conductivity, $\alpha_{i j}^{*}$ : effective coefficients of expansion, $\vec{q}$ : heat flux tensor, $\vec{t}$ : traction tensor)

of the influence of the microstructure. Within the framework of continuum mechanics, it is possible to develop models at the macro level of the material and structural behaviour by introducing effective tensors, which may contain a detailed representation of the microstructure and account for thermomechanical equilibrium on the microlevel. In connection with numerical methods, stress, deformation and damage at thermomechanical loading can be determined for arbitrary structures and boundary value problems. However, little work has been done in this field with respect to refractory materials.

The aim of this study is to present a simple microcrack based damage model for brittle materials under thermo-mechanical dynamical loading conditions. To combine fractureand damage-mechanical approaches, submodels containing a sharp crack tip are introduced in the FEM model at the ends of the damage zones. Within the submodels numerical and analytical approaches can be integrated, representing interactions between macro-cracks and microstructure.
Furthermore, stress intensity factors (SIF) are by calculated using the submodel technique and alternatively the energy release rate is calculated from a conservation line-integral. Results are presented in terms of numerical simulations of damage patterns at different conditions.

\section{Theoretical framework}

We consider a solid continuum with thermomechanical initial and boundary conditions given by stresses $\vec{t}$ and heat flux $\vec{q}$ (Neumann) or displacement $\vec{u}$ and temperature $\theta$ (Dirichlet). To incorporate local microstructural features, mesoscale cell models with linear elastic matrix properties are introduced generally containing voids, cracks or grain boundaries. The cell model with boundary $\partial V$ describes a Representative Volume Element (RVE) [1, 2] in the continuum. In the homogenization process generally we obtain effective elastic $\left(C_{i j k l}^{*}\right)$ and thermal properties $\left(\lambda_{i j}^{*}\right.$ and $\alpha_{i j}^{*}$ ) of an RVE. It is essential that the size of the RVE is chosen according to the condition $L>d>>b$. For brittle materials typical values of an RVE 


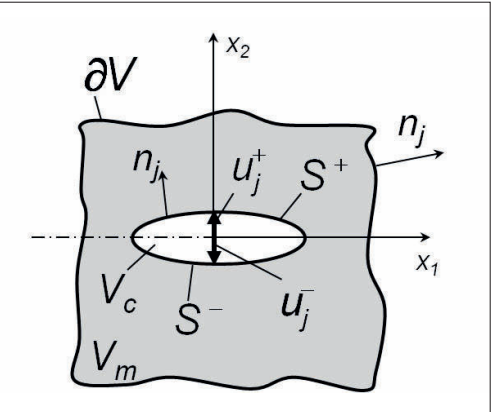

Fig. 2 Defect phase within the matrix volume of an RVE

are $0,1 \mathrm{~mm}$, which is related to the microstructural size scale of real material [3]. The principle procedure of a homogenization process at refractory materials under thermal shock is illustrated in Fig. 1.

To derive average stress and strain tensors for the inhomogeneous field, we consider two subdomains with different properties, i.e. the defect or crack phase with volume $V_{c^{\prime}}$ interface $S$ and unit normal $n_{j}$ inside the matrix material with volume $V_{m}$ and surface $\partial V$, see Fig. 2. The faces of the infinitely thin microcrack are separated according to the positive $\left(x_{2}>0\right)$ and negative $\left(x_{2}<0\right)$ half spaces as $S=S^{+}+S^{-}$. The vector of the displacement jump is defined as:

$\Delta u_{j}=u_{j}^{+}-u_{j}^{-}$

The basic equation for an average macroscopic stress field in a simply connected domain $V$ is given as:

$$
\begin{aligned}
\left\langle\sigma_{i j}\right\rangle= & \frac{1}{V} \int_{V} \sigma_{i j}\left(x_{l}\right) d V=\frac{1}{V} \int_{V}\left(x_{j, k} \sigma_{i k}+x_{j} \sigma_{i k, k}\right) d V \\
& =\frac{1}{V} \int_{V}\left(x_{j} \sigma_{i k}\right)_{k} d V
\end{aligned}
$$

where $x_{j, k}=\delta_{j k}$ and $\sigma_{i k, k}=0$ in the case of quasi static crack growth and without the

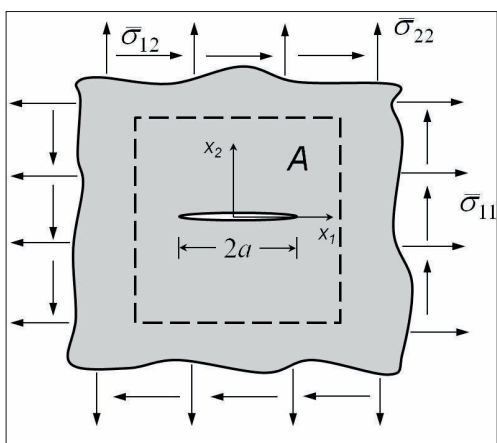

Fig. 3 A single crack in an infinite domain under mixed-mode loading, $A$ is the area of the RVE action of body forces. Applying Gauss's theorem, stress is transformed to the surface. We get the average stress $\left\langle\sigma_{i j}\right\rangle$ with $t_{i}$ as the traction vector and $\partial V$ as the boundary of the RVE:

$\left\langle\sigma_{i j}\right\rangle=\frac{1}{V} \int_{\partial V} x_{j} \sigma_{i k} n_{k} d A=\frac{1}{V} \int_{\partial V} x_{j} t_{i} d A$

For the average stress in both subdomains $V_{c}$ and $V_{m}$ according to Fig. 2 the previous equation leads to:

$$
\begin{aligned}
\left\langle\sigma_{i j}\right\rangle & =\frac{1}{V} \int_{V_{m}} \sigma_{i j}\left(x_{l}\right) d V+\frac{1}{V} \int_{V_{c}} \sigma_{i j}\left(x_{l}\right) d V \\
& =\frac{1}{V} \int_{\partial V} t_{i} x_{j} d A+\frac{1}{V} \int_{S}\left(t_{i}^{c}-t_{i}^{m}\right) x_{j} d A .
\end{aligned}
$$

The $t_{i}^{m}$ and $t_{i}^{c}$ describe the tractions at the boundaries of matrix and defect volumes. Due to continuity of tractions at the interface the last term in equation (4) is disappearing. The equation of volume average macrostrain can according be given as:

$$
\begin{array}{r}
\left\langle\epsilon_{i j}\right\rangle=\frac{1}{2 V} \int_{V}\left(u_{i, j}+u_{j, i}\right) d V= \\
\frac{1}{2 V} \int_{\partial V}\left(u_{i} n_{j}+u_{j} n_{i}\right) d A .
\end{array}
$$

Applying the superposition principle and considering linear elastic behavior for the material matrix, the average strain is decomposed into a part due to the matrix and one due to the defect phase. In case of microcracks of zero stiffness inside the material matrix, equation (5) leads to the result:

$$
\begin{gathered}
\left\langle\epsilon_{i j}\right\rangle=c_{M}\left\langle\epsilon_{i j}\right\rangle_{M}+\frac{1}{2 V} \int_{S^{+}}\left(\Delta u_{i} n_{j}+\Delta u_{j} n_{i}\right) d A \\
=c_{M}\left\langle\epsilon_{i j}\right\rangle_{M}+\left\langle\epsilon_{i j}\right\rangle_{C}
\end{gathered}
$$

with $\left\langle\varepsilon_{i j}\right\rangle_{M}$ as average strain in the surrounding matrix of volume $V_{m}=c_{m} V$ and the displacement jump at the crack interface $S^{-}=S^{+}$according to equation (1). For a defect phase consisting of microcracks, the factor $c_{M}=1$. The last term of equation (6) $\left\langle\varepsilon_{i j}\right\rangle_{C}$ describes the average strain of the defect phase.

Neglecting microcrack interaction, we consider a single crack with initial length $2 a$ in an infinite domain under mixed-mode loading, see Fig. 3.

Mixed-mode loading is considered due to arbitrary crack orientations. However, those cracks are assumed to be most critical, with respect to growth whose faces are perpendicular to the maximum principle stress. The average strain of a microcrack defect phase embedded in an RVE is derived from equation (6):

$\left\langle\epsilon_{i j}\right\rangle_{C}=\frac{1}{2 A} \int_{-a}^{a}\left(\Delta u_{i} n_{j}+\Delta u_{j} n_{i}\right) d x_{1}$

The strain in the $x_{2}$ - direction is obtained from equation (7) as:

$\left\langle\epsilon_{22}\right\rangle_{C}=\frac{1}{A} \int_{-a}^{a} \Delta u_{2} d x_{1}=\bar{\sigma}_{22} \frac{2 \pi a^{2}}{E A}$

and the shear deformation as:

$\left\langle\epsilon_{12}\right\rangle_{C}=\frac{1}{2 A} \int_{-a}^{a} \Delta u_{1}\left(x_{1}\right) d x_{1}=\bar{\sigma}_{12} \frac{\pi a^{2}}{E A}$

with $\Delta u_{i}\left(x_{1}\right)=\frac{4 \bar{\sigma}_{i 2}}{E} \sqrt{a^{2}-x_{1}^{2}} ;(i, j=1,2)[4]$ as displacement jump for Mode-I and Mode-II loading and $E$ as Young's modulus of the matrix material.

In the following, the ratio $\frac{4 a^{2}}{A}=f$ will be introduced as damage variable or crack density parameter. If $f=1$, the microcrack spans the whole RVE, thus the material is locally damaged. The macroscopic average strain of the crack phase according to the equations (8) to (9) becomes:

$\left[\begin{array}{l}\epsilon_{11} \\ \epsilon_{22} \\ \epsilon_{12}\end{array}\right]_{C}=\frac{1}{E}\left[\begin{array}{ccc}0 & 0 & 0 \\ 0 & \frac{f \pi}{2} & 0 \\ 0 & 0 & \frac{f \pi}{4}\end{array}\right]\left[\begin{array}{c}\bar{\sigma}_{11} \\ \bar{\sigma}_{22} \\ \bar{\sigma}_{12}\end{array}\right]$

Equation (10) represents an anisotropic material law for the defect phase. The effective inelastic material law for the damaged material evolves from equation (6) and leads to a generalized Hook's law:

$\left\langle\epsilon_{i j}\right\rangle_{M}+\left\langle\epsilon_{i j}\right\rangle_{C}=\left(C_{i j k l}^{*}\right)^{-1}\left\langle\sigma_{i j}\right\rangle$

Here, $\left(C_{i j k l}^{*}\right)^{-1}$ denotes the effective compliance tensor.

The criterion for microcrack evolution has been chosen in equivalence to a classical R-curve based Mode-I macro crack growth criterion [5]:

$K_{I}(\sigma, a)=K_{R}(\Delta a)$

with $K_{I}$ as Mode-I SIF depending on local stress and the crack length a and $K_{R}$ depending on the crack propagation length a. Considering e.g. a damage zone at the tip of a macroscopic crack (Fig. 4) we have two possible states. If the damage variable $f$ holds the initial value $f_{0}$, the material is isotropic assuming a statistical distribution of orientations of microcracks. If the damage variable is increasing $\left(f>f_{0}\right)$ the material 
becomes anisotropic due to cracks orientated perpendicularly to the direction of principle tensile stress $\sigma_{I}$ growing faster than others. Those are considered relevant and thus dominate in the model for damage regions, see Fig. 4. Therefore, a transformation of the effective elastic tensor $C_{i j k l}^{*}$ with respect to the local crack coordinate system $\left(\bar{x}_{1}, \bar{x}_{2}\right)$ needs to be done. In our continuum damage model, there aren't any macroscopic cracks in terms of free surfaces as depicted in Fig. 4, in fact the crack itself consists of a slender damage zone.

For the isotropic case, microcracks are opened in all directions within the $x_{1}-x_{2}$ plane, thus the applied stresses are:

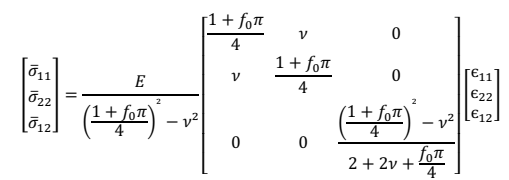

They depend on the initial damage variable $f_{0}$ and the macroscopic strain $\varepsilon_{i j}$. For the anisotropic case the matrix of macro stress is the following:

$\left[\begin{array}{l}\bar{\sigma}_{11} \\ \bar{\sigma}_{22} \\ \bar{\sigma}_{12}\end{array}\right]=\frac{E}{2+f \pi-2 v^{2}}\left[\begin{array}{ccc}2+f \pi & 2 v & 0 \\ 2 v & 2 & 0 \\ 0 & 0 & \frac{2+f \pi-2 v^{2}}{2+2 v+\frac{f \pi}{4}}\end{array}\right]\left[\begin{array}{c}\epsilon_{11} \\ \epsilon_{22} \\ \epsilon_{12}\end{array}\right]$

The essential thermal parameters of refractory materials which influence reliability and life time are thermal conductivity $\lambda_{i j^{\prime}}$ thermal expansion $\alpha_{i j}$ and specific heat $c_{H}$. In this work, hysteresis loops have been modeled for $\alpha(\theta), \lambda(\theta)$ and $c_{H}(\theta)$ covering a tempera-ture range from $20^{\circ} \mathrm{C}$ to $1200^{\circ} \mathrm{C}$. Thermally induced stresses are calculated from Hooke's law introducing the temperature change $\Delta \theta$ :

$$
\sigma_{i j}=C_{i j k l}^{*} e_{k l}^{e l}=C_{i j k l}^{*}\left(\epsilon_{i j}^{t o t}-\alpha_{i j} \Delta \theta\right)
$$

where $\varepsilon_{i j}^{\text {tot }}$ is the total strain and $\varepsilon_{k l}^{e l}$ denotes the elastic strain. In order to simulate thermal stress, the temperature distribution in the material is required. Therefore, the thermal field problem:

$$
\rho c_{H} \frac{\partial \theta}{\partial t}=\lambda_{i j} \frac{\partial^{2} \theta}{\partial x_{i} \partial x_{j}}
$$

is solved first, supplying a transient temperature field as loading quantity for the mechanical boundary value problem.

To describe interactions between a damage zone representing a macroscopic crack and the microstructure it is possible to apply a submodel technique. Since a tip of a damage zone does not exhibit a singularity, it is feasible to include a sharp crack tip in a submodel, which is introduced in the global model at the end of the damage zone. Thus, fracture- and damage-mechanical approaches are combined in only one numerical FEMsimulation (Fig. 5). In general, there is a closure effect due to a finite stiffness at the integration points belong to the damage zone, which can be illustrated as spring elements between crack faces. This effect can be observed by experimental analysis of thermal shock.

Another method to determine the stress intensity factors is to calculate the J-integral. A commercial implementation cannot be applied here, since there are no real crack faces in terms of free surfaces. Thus, a line integral is calculated with an integration contour reaching from one boundary of the damage zone to the other. For a Mode-I loading with

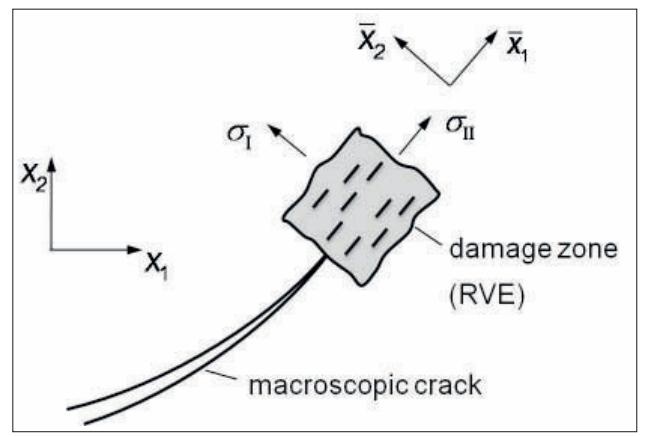

Fig. 4 Schematic representation of a damage zone with equally distributed parallel cracks inside the RVE and local and global coordinate systems

the direction of crack extension $z_{k}$, the SIF evolves from [5]:

$K_{I}^{2}=\frac{E z_{k}}{1-v^{2}} \int_{S}\left(\frac{1}{2} \sigma_{m n} \varepsilon_{m n} n_{k}-t_{i} u_{i, k}\right) d S$

Crack surface integrals cannot be introduced, so results of equation (17) are path dependent.

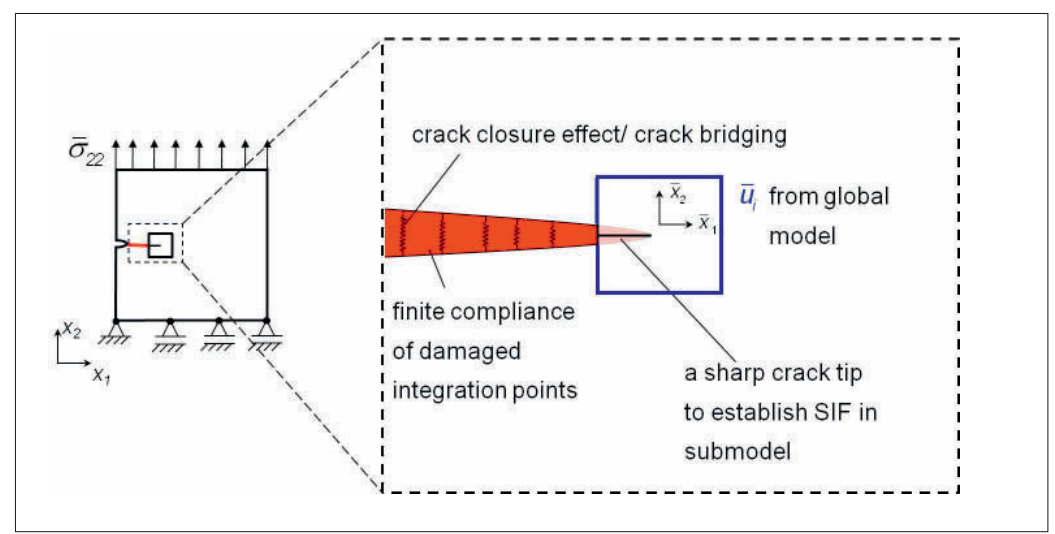

Fig. 5 Global model under tensile load (left) and damage zone with crack tip submodel and displacement boundary condition $\bar{u}_{i}$ from global model (right)

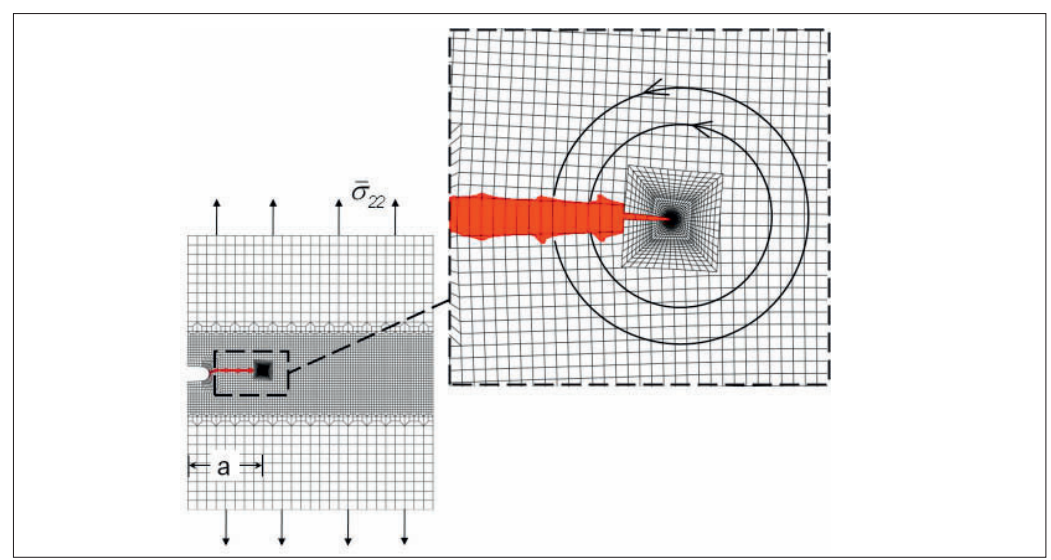

Fig. 6 Damage zone at Mode-I loading $\left(\bar{\sigma}_{22}=96 \mathrm{MPa}\right)$ with crack tip submodel and integration contours 


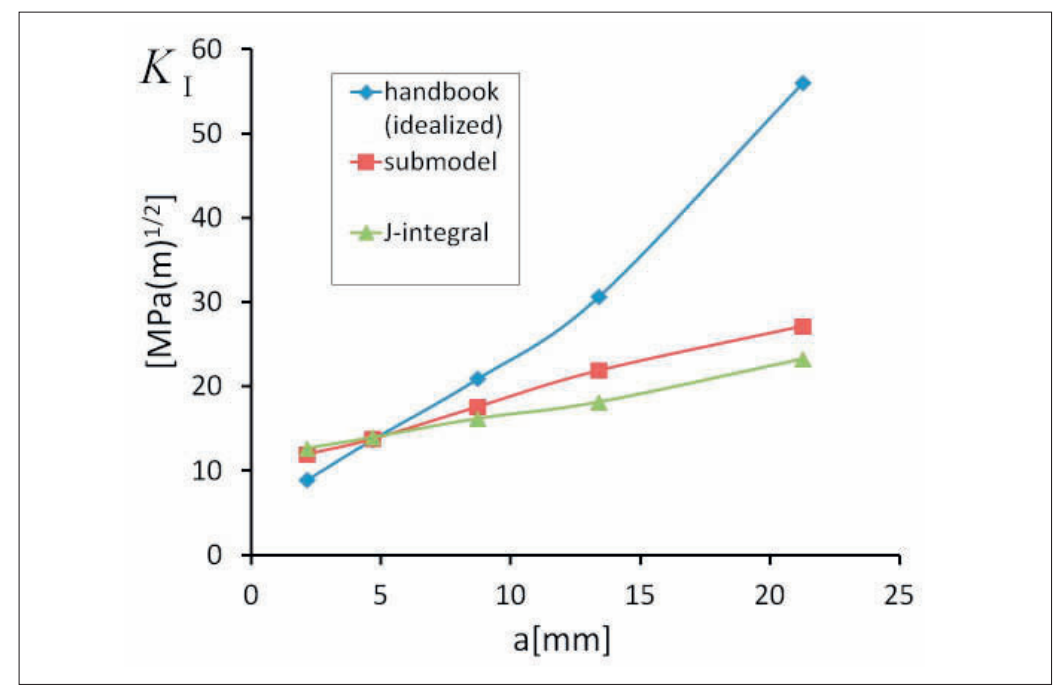

Fig. 7 SIF at Mode-I loading (see Fig. 6) calculated by three different methods

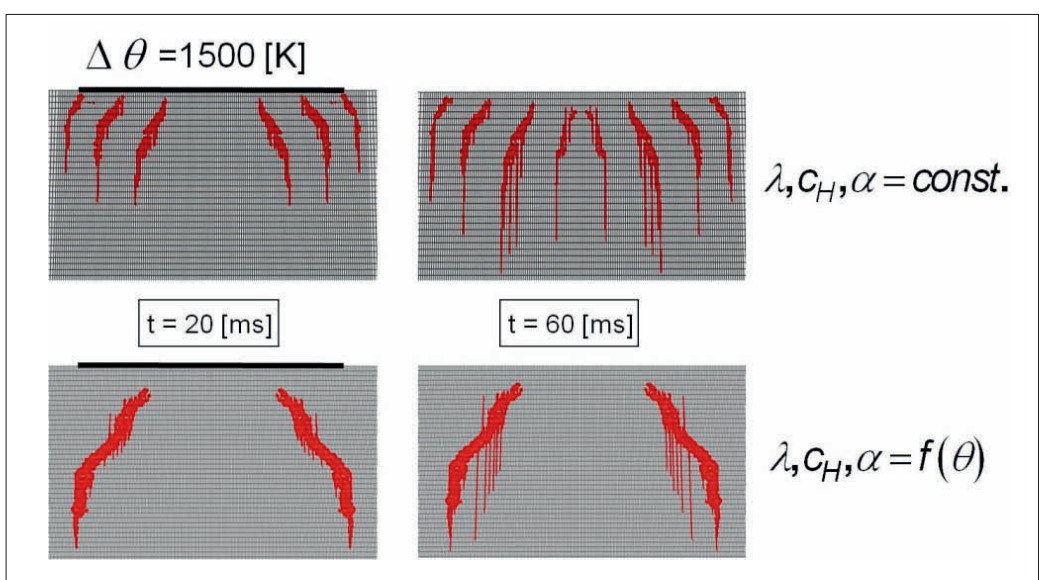

Fig. 8 Damage patterns at thermal shock $\Delta \theta=1500 \mathrm{~K}$ after 20 and $60 \mathrm{~ms}$ with and without temperature dependent properties

\section{Numerical examples}

The anisotropic, non-linear material law is implemented into the Finite Element Code ABAQUS using a Subroutine UMAT. Fig. 6 shows the FE-model of a plate with tensile loading $\bar{\sigma}_{22}$. The damage zone initiating at the notch is growing like a macroscopic crack of length a. At the tip of the damage zone the submodel and two integrations the handbook solution for an ideal crack. Whereas bridging is taken into account at the damage model via finite stiffness of the damage zone, the handbook solution is based on traction-free crack surfaces, thus leading to much higher values of $K_{I}$.

As a second test geometry, we take a plate with temperature jump $\Delta \theta=1500 \mathrm{~K}$ at the top surface, Fig. 8. Of course, thermal shock simulations have to account for temperaturedependent material data and inertia effects. In a simulation with constant param-eters we observe equally spaced crack nucleation starting close to the surface. In the simulation with temperature dependent par-ameters $\lambda(\theta), c_{H}(\theta)$ and $\alpha(\theta)$ the crack initiation starts underneath the surface. In any case damage zones are initiated at locations with highest temperature and stress gradients.

\section{Summary}

A continuum damage model for refractories ceramics is presented incorporating fracture mechanical approaches. Results shows damage patterns under thermal shock conditions.

\section{Acknowledgment}

Financial support by the German Science Foundation (DFG) within the SPP 1418 is gratefully acknowledged.

\section{References}

[1] Qin, Q.-H.; Yang, Q.-S.: Macro-micro theory on multifield coupling behavior of heterogeneous materials. Berlin, Heidelberg, New York 2008, chap. 2

[2] Hill, R.: Elastic properties of reinforced solid. J. Mech. Phys. Solids 11 (1963) 357-372

[3] Dormieux, L.; Kondo, D.; Ulm, F-J.: Microporomechanics. New York 2006

[4] Gross, D.; Seeling, Th.: Bruchmechanik. 4th Ed. Berlin, Heidelberg, New York 2007

[5] Kuna, M.: Numerische Beanspruchungsanalyse von Rissen. Wiesbaden 2008 